\title{
Droplet Evaporation and De-pinning in Rectangular Microchannels
}

\author{
A. Odukoya ${ }^{1}$ and G. F. Naterer ${ }^{2}$ \\ ${ }^{1,2}$ University of Ontario Institute of Technology, Oshawa, Ontario, Canada
}

\begin{abstract}
Experimental and numerical studies are presented for evaporation of micro-droplets of deionised (DI) water and toluene on lead zirconate titanate (PZT) substrates. The microchannels are fabricated with SU-8 2025 and 2075. The effects of channel width and depth on the evaporation and de-pinning rates of embedded micro-droplets are presented and compared for both fluids. The studies indicate a partially hydrophobic nature of SU-8/PZT microchannel to DI water and complete wetting when toluene is used as the droplet. The rate of evaporation of toluene is about double the rate of evaporation of DI water. Comparisons of the rates of evaporation and de-pinning show that the channel width has a larger effect on evaporation than the depth of the channel. The equivalent contact angle of the pinned film and bulk fluid compensated for the evaporation of the droplet. Surface roughness was also shown to have a significant effect on the pinned film in the rectangular microchannels.
\end{abstract}

\section{Nomenclature}

A $\quad$ cross sectional area $\left(\mathrm{m}^{2}\right)$

C vapor molar concentration $\left(\mathrm{mol} / \mathrm{m}^{3}\right)$

$D_{A B} \quad$ diffusion coefficient $\left(\mathrm{m}^{2} / \mathrm{s}\right)$

$e_{v} \quad$ uncertainty in voltage measurement (V)

\footnotetext{
${ }^{1}$ Research Associate, Faculty of Engineering and Applied Science, University of Ontario Institute of Technology, 2000 Simcoe Street North, Oshawa, Ontario, Canada, L1H 7K4

${ }^{2}$ Dean, Faculty of Engineering and Applied Science, Memorial University, St. John's, Newfoundland, 240 Prince Phillip Drive, St. John's, NL Canada A1B 3X5
} 


\begin{tabular}{|c|c|}
\hline$e_{i}$ & uncertainty in current measurement (A) \\
\hline $\mathrm{ep}_{\mathrm{p}}$ & uncertainty in pixel measurement \\
\hline$e_{w}$ & uncertainty in width measurement (m) \\
\hline$e_{F_{N}}$ & uncertainty in frame number \\
\hline$e_{F_{R}}$ & uncertainty in width measurement (m) \\
\hline $\mathrm{F}_{\mathrm{N}}$ & frame number \\
\hline$F_{R}$ & frame rate \\
\hline $\mathrm{g}$ & acceleration due to gravity $\left(\mathrm{m} / \mathrm{s}^{2}\right)$ \\
\hline h & heat transfer coefficient $\left(\mathrm{W} / \mathrm{m}^{2} \mathrm{~K}\right)$ \\
\hline j(w) & evaporation rate per unit area $\left(\mathrm{kg} / \mathrm{m}^{2}\right)$ \\
\hline l & thickness (m) \\
\hline $\mathrm{L}$ & length (m) \\
\hline $\mathrm{M}_{\mathrm{D}}$ & mass (kg) \\
\hline $\bar{M}$ & molecular weight (kg/kmol) \\
\hline $\mathrm{P}$ & pressure $\left(\mathrm{N} / \mathrm{m}^{2}\right)$ \\
\hline $\bar{R}$ & universal gas constant (J/kmol K) \\
\hline$t$ & time (s) \\
\hline$T$ & temperature $\left({ }^{\circ} \mathrm{C}\right.$ or $\left.\mathrm{K}\right)$ \\
\hline$\overline{\mathrm{u}}$ & instantaneous velocity $\left(\mathrm{m} / \mathrm{s}^{2}\right)$ \\
\hline $\mathrm{U}$ & uncertainty \\
\hline
\end{tabular}




$\begin{array}{cl}\mathrm{V} & \text { volume }\left(\mathrm{m}^{3}\right) \\ \mathrm{w} & \text { width of microchannel }(\mathrm{m}) \\ \mathrm{W}_{\mathrm{o}} & \text { initial width }(\mathrm{m}) \\ \mathrm{Z}_{\mathrm{n}} & \text { distance from neutral axis (m) }\end{array}$

\section{Greek}

$\begin{array}{ll}\Delta & \text { discrete change, or difference } \\ \mu & \text { dynamic viscosity }(\mathrm{kg} / \mathrm{m} \mathrm{s}) \\ \rho & \text { density }\left(\mathrm{kg} / \mathrm{m}^{3}\right) \\ \theta & \text { contact angle }\left(^{\circ}\right) \\ \phi & \text { concentration gradient of evaporating fluid }\left(\mathrm{mol} / \mathrm{m}^{3}\right) \\ \bar{\sigma} & \text { accommodation coefficient }\end{array}$

\section{Subscripts}

air air

app apparent

c critical contact angle

D droplet

e experiment

$f \quad$ friction without roughness

i initial

L left

lv liquid vapour interface 


\begin{tabular}{|c|c|}
\hline $\mathrm{m}$ & meniscus \\
\hline 0 & start \\
\hline $\mathrm{R}$ & right \\
\hline sub & substrate \\
\hline $\mathrm{t}$ & total \\
\hline $\mathrm{v}$ & vapor \\
\hline $\mathrm{W}$ & water droplet \\
\hline \multicolumn{2}{|c|}{ Abbreviations } \\
\hline CIRFE & Center of Integrated Radio Frequency Engineering \\
\hline DI & Deionized \\
\hline IPA & Isopropyl Alcohol \\
\hline LOR & Lift Off Resist \\
\hline MEMS & Microelectromechanical System \\
\hline MHE & Micro Heat Engine \\
\hline $\mathrm{PZT}$ & Lead Zirconate Titanate \\
\hline RCA & Radio Cooperation of America \\
\hline $\mathrm{RF}$ & Radio Frequency \\
\hline RIE & Reactive Ion Etching \\
\hline UV & Ultra Violet \\
\hline
\end{tabular}

\section{Introduction}

Various ways have been invented and commercialized to harness energy from water, such as hydroelectricity, tidal energy, among others. This paper investigates transport phenomena of micro-droplets in micro-electromechanical systems (MEMS) for powering micro-devices. MEMS 
generally require small quantities of energy, so generating electricity for their use can reduce their dependence on conventional power generating systems such as batteries. Increasing the efficiency of micro-power generating systems embedded in MEMS devices can increase their length of operation. For example, heat recovery by electrical components in MEMS devices can be used to generate voltages that power individual electrical components within microchips.

MEMS heat recovery can be achieved by various methods such as thermoelectric or piezoelectric materials in MEMS devices. Thermoelectric materials have the ability to convert heat directly to electricity, while piezoelectric materials can convert stresses in a material into electrical voltages. This paper examines the transport phenomena associated with thermal energy conversion for MEMS devices, in particular for a Micro Heat Engine (MHE). Earlier investigations [1, 2] showed that surface tension of water can be used for thermocapillary pumping in a microchannel. An MHE can use thermocapillary effects to induce stresses in a piezoelectric membrane by the cyclic heating and cooling of a droplet in a microchannel [1 - 4].

When heat is applied to one end of the droplet in a closed microchannel, an increase in temperature at the heat source leads to a temperature gradient across the droplet. This results in a displacement of the droplet along the length of the channel towards the cooler end of the channel. This process is known as thermocapillary pumping. The resulting displacement increases the pressure at the closed end of the channel. The increased pressure is used to induce stress on a membrane at the closed end of the channel. This induced stress results in mechanical deformation of the membrane. The mechanical deformation of the membrane then leads to a flow of electrons in an externally connected circuit, which yields conversion of mechanical energy to electrical energy. Fig. 1 shows a schematic of the MHE and the forces acting within the microchannel. 
Past studies [5] have conflicting information about the behaviour of water droplets on the micro/nano-scale. This paper presents new experimental and analytical results that provide better understanding of evaporation of deionized water (DI-water) and toluene in SU-8 microchannels. The SU-8 microchannels are fabricated on lead zincronia titanate (PZT) substrates. To the best of our knowledge, there is little or no available information on the transport phenomena associated with water or toluene droplets on PZT substrates, which are used for thermocapillary pumping in the MHE. This paper presents new data regarding these transport phenomena of water microdroplets in rectangular microchannels.

Better understanding of transport phenomena associated with evaporation of droplets is necessary for successful fabrication of droplet-based MHEs. Evaporation is a complex process to predict due to various processes, which are associated with heat and mass transfer occurring simultaneously. A comprehensive review of different phenomena associated with evaporation was conducted by Bonn et al. [5]. Experimental and numerical analysis of heat and mass transfer involving the contact line displacement was investigated. The main focus was the liquid-vapor contact line relative to the vapor-solid contact line, as well as relative to the substrate-liquid contact line. The initial spreading of liquids on a solid substrate has been analysed by the Gibbs adsorption equation to estimate the change in surface tension [6]. In order to predict the dynamics of the evaporation, the hydrodynamics of droplet spreading must be well understood. Other studies in past literature have examined droplet spreading on a flat surface [7 - 13].

This paper investigates droplet spreading in a rectangular microchannel. Past studies have generally assumed a spherical droplet $[7,9,11,14]$, but with the existence of a film, which is 
detached from the main surface. There are two major ways in which evaporation occurs on a flat surface. Firstly, the droplet radius remains approximately constant, while the contact angle of the liquid-substrate contact angle decreases [14]. Secondly, the other method of evaporation occurs where the contact radius decreases while the contact angle remains constant [14]. In the latter case, both the contact angle and contact radius decrease simultaneously at a certain contact radius. This stage precedes the de-pinning process. The de-pinning process occurs when the film starts to evaporate and appears to be separate from the main droplet. The separation of these two layers of the liquid was used to transfer solid particles in a liquid droplet to a substrate in a previous study by Deegan [10]. This is the technology used for inkjet printers [15] as the carrier liquid evaporates and patterns are deposited on the paper. Deegan [15] used this transport phenomenon to explain the pattern of satins deposited by a drop of coffee. The shape of pinned polymer films is dependent on the pinning time and distance between the bulk fluid and pinned film [16].

The physical processes associated with evaporation also occur due to the nature of the droplet during evaporation. The boundary of the surface can be either saturated with vapor or a mixture of liquid and vapor of unknown proportions [17]. A temperature change within the droplet occurs as a result of evaporation, but it is generally difficult to estimate the magnitude of heat transfer to the substrate or the atmosphere as the process is occurring at atmospheric conditions. This also leads to internal recirculation within the droplet caused by a change in surface tension $[18,19]$.

The flow within the droplet occurs towards the edge of the droplet so as to maintain a constant volume. The constant change in contact angle leads to a variation in the advancing and receding contact angles. Various methods have been proposed for estimating the varying contact angles [20, 21]. A method of estimating the change in contact radius with respect to contact angle 
is a semi empirical approach proposed by Deegan et al. [14]. The authors developed a method by assuming an equivalent contact angle, based on the change in volume of the droplet and proportional to an exponential function of time [14]. Measured results from experiments conducted by Shahidzadeh [12] showed that the vapor above the droplet accounts for the variation in the exponent. Different liquids will lead to different exponents in relation to time, i.e., $R\left(t-t_{0}\right)^{\lambda}$, where $\lambda$ varies for different liquids with respect to the equivalent contact angle. Here $R$ is the contact radius, $t$ is the time for the droplet of a specified volume to evaporate, and $t_{0}$ is the reference time.

The behaviour of wetting and non-wetting surfaces differs during evaporation. Cahcile et al. [8] developed a semi-empirical correlation for estimating the hydrodynamic behaviour of alkanes on a wetting surface. The analysis neglected the pinning of the droplet on the substrate. The assumption of a spherical shape of the droplet appeared to be an over-simplification of the problem. It was recommended that an electrostatic model including the pinning process should be included in the predictive formulation.

In this paper, a new formulation of droplet evaporation and de-pinning in rectangular microchannels will be presented. The problem formulation is described in section 2, as well as the numerical formulation of the evaporation and de-pinning processes. Section 3 describes the fabrication of the closed rectangular microchannel and the experimental setup. Experimental and numerical results are presented and discussed in section 4 .

\section{Problem Formulation}

The evaporation of a droplet in a rectangular channel has three major thermophysical processes, namely mass transfer of the bulk fluid into the gas phase, heat transfer as a result of natural convection and Marangoni convection due to temperature gradients. In this paper, the mass 
transfer and natural convection are assumed to be the major transport phenomena that affect the evaporation of the droplet. Heat addition was not implemented in the current experimental studies, hence the temperature gradients are neglected during the evaporation process.

\subsection{Droplet evaporation model}

When a droplet is injected into a microchannel, it takes the shape of the channel. This process is dependent on the wetting properties of the fluid. The droplet can either have a concave or convex meniscus with respect to the substrate of the channel. If the droplet is much smaller than the channel dimensions, it will adhere to the edge of the channel. The model in this paper is developed for the hydrophobic case, as per the experiments. The model can also be extended to the hydrophilic case, since the main difference is the contact angle and volume associated with the concave meniscus. Consider the cross-section of the channel and droplet shown in Fig. 2.

The transient mass transfer diffusion equation can be used to express the rate of evaporation of the droplet into the surrounding vapor. This can be expressed based on Fick's law of diffusion as follows,

$\frac{\partial \rho_{D}}{\partial t}=D_{A B} \frac{\partial^{2} \rho_{D}}{\partial w^{2}}$

subject to the following boundary conditions: $\rho_{D}=\rho_{w}$ at $t=0$ for $0 \leq w \leq w_{o}$, (at the beginning of the evaporation, the density at the surface is equivalent to the initial density of the droplet injected into the channel) $\rho_{D}=\rho_{\text {air }}$ at $w=0$ for $\mathrm{t}>0$, (at the end of the evaporation cycle, the density at the surface is equivalent to the density of the surrounding air at the channel surface) and $\rho_{D}=\rho_{w}$ at $w=w_{o}$ for $\mathrm{t}>0$, (during evaporation, the density at the surface is assumed to be

equivalent to the density of the droplet, while the mass transfer is estimated based on the volume 
of the droplet). Also, $w_{o}$ is the initial width of the droplet, $\rho_{D}$ is the density adjacent to the surface, $\rho_{\text {air }}$ is the density of air, and $\rho_{w}$ is the density of water. Non-dimensionalizing the mass transfer equation and using separation of variables, the change in density with time can be expressed as $\rho_{D}(\mathrm{t})=\frac{4 \mathrm{Y}_{0}\left(\rho_{\mathrm{w}}-\rho_{\text {air }}\right)}{(2 \mathrm{n}+1) \pi} \sum_{\mathrm{n}=0}^{\infty}[\sin (\lambda w)] e^{-D_{A B}\left(\frac{2 \mathrm{n}+1}{L}\right)^{2} t}+\rho_{\text {air }}$

where $Y=\frac{\rho_{D}-\rho_{\text {air }}}{\rho_{w}-\rho_{\text {air }}}$ and the boundary conditions become $Y=Y_{0}=1$ at $t=0$ for $0 \leq w \leq w_{o}$, (density is equivalent to the density of water), $Y=0$ at $w=0$ for $t>0$, (all bulk fluid has evaporated) and $Y=Y_{0}=1$ at $w=w_{o}$ for $\mathrm{t}>0$. Details of the expansion and solution can be found in Appendix A.

From the mass transfer equation, the change in volume with time can be written as

$$
\frac{d V}{d t}=-\frac{D_{A B}}{\rho_{w}} \int_{A} \frac{d \rho_{D}}{d w} \cdot d A
$$

Since the cross-sectional area is assumed to be constant as the droplet evaporates along the width of the channel, the rate of evaporation relative to the width of the droplet can be obtained from a numerical integration over the channel width as follows.

$$
w(t)=-\frac{D_{A B}}{\rho_{w}} \int\left[\frac{4 \mathrm{Y}_{0}\left(\rho_{\mathrm{w}}-\rho_{\text {air }}\right)}{L} \sum_{\mathrm{n}=0}^{\infty}\left[\cos \left(\frac{2 n+1}{L} \pi w\right)\right] e^{-D_{A B}\left(\frac{2 n+1}{L}\right)^{2} \pi^{2} t}\right] d t
$$

Equation (4) is used to predict the change in width of the droplet with time, assuming the droplet spans across the entire width of the channel. The Hertz-Knudsen-Scharge equation [22] is used to estimate the effect of natural convection which occurs during evaporation of the meniscus. The temperature of the droplet is assumed to be equal to the interfacial liquid temperature at the interface with air, 


$$
\frac{\partial m}{\partial t}=A\left[\frac{2 \bar{\sigma}}{2-\bar{\sigma}}\left(\frac{\bar{M}}{2 \pi \bar{R}}\right)^{1 / 2} \frac{1}{T_{l v}^{1 / 2}}\left(P_{v e}\left[T_{l v}\right]-P_{v}\right)\right]
$$

where $\bar{\sigma}$ is the accommodation coefficient, $\bar{M}$ is the molecular weight of the droplet, $\bar{R}$ is the universal gas constant, $T_{l v}$ is the interfacial liquid temperature and $P_{v e}$ is the equilibrium vapour pressure. Coupling equation (1) with the vapour transport equation,

$$
-\frac{\partial C_{v}}{\partial w}=A\left(\frac{1-\frac{C_{v}}{C_{\text {total }}}}{M \cdot D_{A B}}\right)\left[\frac{2 \bar{\sigma}}{2-\bar{\sigma}}\left(\frac{\bar{M}}{2 \pi \bar{R}}\right)^{1 / 2} \frac{1}{T_{l v}^{1 / 2}}\left(P_{v e}\left[T_{l v}\right]-P_{v}\right)\right]
$$

where $\mathrm{C}_{\mathrm{v}}\left(\frac{P_{v}}{P_{\text {total }}} C_{\text {total }}\right)$ and $\mathrm{C}_{\text {total }}\left(\frac{P}{\bar{R} T_{l v}}\right)$ are the vapor mole concentration and total gas mole number per $\mathrm{m}^{3}$ of the evaporated droplet. Integrating equation (6) will yield the effect of convection on the change in width of the droplet. Combining equations (1) and (6), the combined effect of diffusion and convection of the vapor from the surface will give the total effect of evaporation on the width of the droplet.

The experimental results show that a thin layer exists between the main part of the droplet and the wall. This layer is called the "pinned film", which starts to evaporate after the main portion of the droplet has evaporated.

\subsection{Droplet de-pinning model}

A thin film of the droplet is attached to the bottom of the channel and it remains distinct from the bulk of the droplet. This separation occurs along the contact line of the main droplet. The process by which this layer evaporates is known as "de-pinning”. Unlike the evaporation process, the de-pinning process starts from the center of the channel and progresses in both directions simultaneously towards the wall. Fig. 3 shows the de-pining process. The phenomenon is a 
complex process to predict accurately. It occurs in a similar way as evaporation. A semi-empirical approach will be used to analyse the evaporation of the pinned film. The conservation equation of the pinned film can be written as

$\frac{\partial w}{\partial t}+\rho A \frac{\partial}{\partial w}(\bar{u})=-j(w, t)=-D_{A B} \nabla \phi$

where w is the width of the channel, $D_{A B}$ is the diffusivity, $\phi$ is the concentration and $j(w, t)$ is the evaporation rate per unit area. The equation of the average velocity over the width can be expressed as

$\bar{u}(w, t)=-\frac{1}{\rho A} \int_{0}^{w_{o}}\left(\frac{\partial x}{\partial t}+j(w, t)\right) d w$

The evaporation rate of the droplet has been predicted by Deegan [15], Cachile et al. [8] and Poulard et al. [11] to be proportional to the change in pinning radius based on the instantaneous time and final time. It can be represented as

$$
j(w, t)=j_{o}\left(w_{o}-w(t)\right)^{-\kappa}
$$

where

$\kappa=\frac{\pi-2 \theta_{c}}{2 \pi-2 \theta_{c}}$

Here $\theta_{c}$ is the contact angle of the droplet and the contact line at the beginning of the de-pinning process. Also, $j_{o}$ is the evaporation rate at $w=w_{o}$. The volume of the pinned film can be estimated as the volume of the spherical cap of the droplet and the cuboid along the channel length. Extending 
the equations (7) - (10) to the case of pinning in a rectangular microchannel, the expression can be written for the change in volume with time as

$$
\frac{d V}{d t}=\frac{d}{d t}(w y z)+\frac{d}{d t}\left[\frac{\pi w^{3} \theta_{c}}{4}\right]
$$

Here $w, y$, and $z$ are the width, length and depth of the film, respectively. The first term on the right side of equation (11) is the volume of the film along the channel length and the second term is the volume of the spherical cap. Since $\theta_{c}$ is assumed to be constant, based on the particular wetting fluid, and the cross-sectional area $(y z)$ is also constant, the expression can be reduced to

$$
\frac{d V}{d t}=y z \frac{d w}{d t}+\frac{\pi \theta}{4} \frac{d w^{3}}{d t}
$$

Since the evaporation rate can also be related to mass transfer across the boundary, the change in volume becomes

$$
\frac{d m}{d t}=A \cdot\left(w_{o}-w(t)\right)^{-\kappa}
$$

Combining equations (12) and (13), the change in pinning radius becomes

$$
w(t)=\int \frac{A}{\rho}\left(\frac{\left(w_{o}-w(t)\right)^{-\kappa}}{y z+\frac{3 w_{o} \pi \theta}{4}}\right) d t
$$

An expression is determined for $w(t)$ in order to integrate the result over a known time interval and determine the change in the pinning radius. A method of asymptotic analysis will be 
used to find $w(t)$, by an assumption based on observations of the experiment for the profile of the pinning displacement. From the experimental observations, $w(t)$ is determined based on a correlation as follows:

$$
w(t)=\frac{w_{o}}{2}\left(t_{f}-t_{i}\right)^{\kappa}
$$

The value of $\kappa$ is dependent on the type of fluid. A value of 0.3 was used for DI-water and a value of 0.6 was used for toluene, based on a similar asymptotic analysis performed previously by Cachile et al. [8].

The next section will decribe the experimental setup and procedures to investigate the evaporation and de-pinning process in the rectangular microchannel. Detailed steps on the fabrication techniques will be outlined and an uncertainty analysis of the experimental measurements will be presented.

\section{Experimental Apparatus and Procedure}

Fabrication steps of the MHE are illustrated in Fig. 4. The MHE is fabricated with PZT wafers. Three sets of wafers of different thicknesses were examined for the fabrication $(100 \mu \mathrm{m}$, $80 \mu \mathrm{m}$ and $60 \mu \mathrm{m})$.

\subsection{Fabrication of rectangular microchannel}

The PZT substrates of different thicknesses were cleaned with an RCA 1 cleaning procedure. The cleaned substrate was then immersed in a beaker filled with DI-water and allowed to cool for three minutes. High pressure nitrogen gas was used to dry the substrates. Extra care must be taken to ensure the substrates do not fracture in the process. It was difficult to completely 
dry the fragile substrate; hence before proceeding to the next step, the substrate was heated to $120^{\circ} \mathrm{C}$ for two minutes on the vacuum hot plate and allowed to cool.

Photolithography was used to fabricate the SU-8 microchannel. The photoresist was deposited on the substrate and spin coated at 500rpm for $10 \mathrm{~s}$ with an acceleration of $162 \mathrm{~m} / \mathrm{s}^{2}$, $2000 \mathrm{rpm}$ for $15 \mathrm{~s}$ with an acceleration of $468 \mathrm{~m} / \mathrm{s}^{2}$, and $4000 \mathrm{rpm}$ for $20 \mathrm{~s}$ acceleration of $468 \mathrm{~m} / \mathrm{s}^{2}$ in one spin cycle. Edge ridges were observed after the spin cycle. A knife edge was used to remove the ridges from sides of the substrate. Soft baking was performed in two steps for both types of SU-8. The baking time for SU-8 2025 was two minutes at $65^{\circ} \mathrm{C}$ and five minutes at $95^{\circ} \mathrm{C}$, while the baking time for SU-8 2075 was three minutes at $65^{\circ} \mathrm{C}$ and seven minutes at $95^{\circ} \mathrm{C}$. After the baking was completed, the sample was suspended in air for one minute before it was placed on clean wipes on the work bench and allowed to cool naturally. The exposure time for SU-8 using the UV mask aligner was 240s.

Once the exposure was complete, the post exposure baking was commenced. SU-8 2025 was post baked in two steps, one minute at $65^{\circ} \mathrm{C}$ and five minutes at $95^{\circ} \mathrm{C}$. SU-8 2075 was baked for two minutes at $65^{\circ} \mathrm{C}$ and seven minutes at $95^{\circ} \mathrm{C}$. Development was completed after the sample was cooled. SU-8 Developer form Microchem was used to process the pattern after post exposure baking. The optimal development time for SU-8 2025 was four minutes, while that of SU-8 2075 was six minutes. The sample was then immersed in a solution of IPA for three minutes to remove any trace of the developer on the substrate. High pressure nitrogen gas was used to dry the substrate. The fabricated channel was then inspected under a microscope. Fig. 5 shows the fabricated microchannel. 


\subsection{Experimental measurement and uncertainty}

A $10 \mu \mathrm{l}$ syringe was used to dispense the droplet into a microchannel with the aid of a stereo microscope in the experimental measurements. Figure 6(a) shows PZT is partially hydrophobic to the water droplet and a portion of the droplet pinned to the substrate, while the hydrophobic surface contains the bulk fluid. A digital video recorder was mounted on the viewing port of the microscope. The images were recorded at a frequency that varied between 25 and 32 frames per second (fps). The DI-water samples were measured mostly at 25fps because the evaporation occurred slower than toluene. The images were measured under two magnification settings $(\times 5$ and $\times 10$ ), although the microscope had a capability of measuring a magnification of $\times 150$. The entire width of the channel was captured by the digital camera under the latter two settings. The evaporation and de-pinning processes of the droplet were recorded by Stream Pix III imaging software, which allows for digitization and characterisation of the images. The contact angle, droplet width, and pinning radius were measured over time. An imaging tool was used to measure pixel displacement and contact angle over the evaporation and de-pinning periods. The imaging tool also reported the mean and standard deviation of the measurements. This standard deviation was used to estimate the uncertainty of the measured data.

The uncertainty of the measurements was determined based on the Kline and McClintock method [23]. The displacement is a product of the measured width of the channel and the number

of pixels ( $L=P_{i} W$ ). In this case, the independent variables were width and pixels. The uncertainty wasestimated as follows:

$$
U_{L}=\left[\left(\frac{\partial L}{\partial P_{i}} e_{p_{i}}\right)^{2}+\left(\frac{\partial L}{\partial W} e_{w}\right)^{2}\right]^{1 / 2}
$$


where $U_{L}$ is the uncertainty of a single measurement of the length and $e_{p_{i}}, e_{w}$ are the uncertainties associated with measuring the pixel and width, respectively. The uncertainty associated with measuring the evaporation width was estimated as between 1 and $11 \%$ for the experiments considered in this study. The uncertainty associated with the de-pinning process varied between 1 and 5\%. Although some of the data points for the evaporation uncertainty measurements fell outside of the confidence interval of $10 \%$, the single sample uncertainty measurement allowed this set of data to be discarded during the analysis of the experimental data.

The uncertainty in the time measurement is a function of the frame rate and number of frames. The time $t$ was estimated as

$$
t=F_{N}\left(F_{R}\right)^{-1}
$$

The uncertainty in measuring time for a single sample can be expressed as $U_{t}=\left[\left(\frac{\partial t}{\partial F_{N}} e_{F_{N}}\right)^{2}+\left(\frac{\partial t}{\partial F_{R}} e_{F_{R}}\right)^{2}\right]^{1 / 2}$

where $e_{F_{N}}, e_{F_{R}}$ are the uncertainties in measuring the frame number and frame rate, respectively. The uncertainty of the time measurement was found to be about $1 \%$, which is reliable for analysing the experimental data. A high accuracy was expected since the Stream Pix software acquires images at $+/-0.0001$ frame rates per second.

The following section highlights results from the analytical modeling and experimental results. Experimental results will be compared with the predicted models to validate the analytical modeling. 


\section{Results and Discussion}

Experimental and analytical results of the evaporation and de-pinning of the toluene and DI-water will be reported in this section. Earlier investigations by Glockner and Naterer [1, 2] suggested that two promising fluids for thermocapillary pumping were toluene and water. Toluene is completely wetting when injected into the SU-8 channel. DI-water was used for this experiment to avoid the effects of the electric double layer caused by charges present at the wall in the case of regular water.

DI-water is partially wetting when injected into the microchannel, making the surface partially hydrophobic in this test case. The pattern of evaporation of DI-water and toluene is shown in Figs. 6 and 7 respectively. When DI-water is injected into the microchannel, the bulk fluid is separated from the pinned film as shown in Fig. 6(a), due to the hydrophobic nature of the SU-8 vertical walls to the fluid. After about $0.92 \mathrm{~s}$, the bulk fluid spreads across the width of the channel, reducing the separation distance between the bulk fluid and pinned film as shown in Fig. 6(b), due to evaporation of the bulk fluid. In order to maintain constant volume, the droplet spreads along the width of the microchannel, while preserving the contact angle with the surface. After about 3.32s (Fig. 6(c)), the rate of change in width of the bulk fluid decreases significantly, when comparing the change in width with that observed in Fig. 6(d), which represents the end of the evaporation of the bulk fluid.

The evaporation of the bulk fluid in the case of toluene evolves through a different pattern since it is completely wetting the PZT substrate and SU-8 vertical walls. Fig. 7(a) further establishes the latter claim where the pinned film is not visible. After about 0.76s (Fig. 7(b)), the pinned film can be observed, indicating a separation between the pinned film and bulk fluid. The evaporation of the bulk fluid initially occurs only across the width, but after about 2.76s, the bulk 
fluid starts to evaporate along the length as shown in Fig. 7(c). The complete evaporation of the bulk fluid occurs after about 3.76s as observed in Fig. 7(d).

The results of non-dimensional width and a comparison of the evaporation of DI-water and toluene are shown in Fig. 8. Toluene evaporates faster than water, but exhibits similar trends as observed in Fig. 8. The analytical model yields the correct trend of evaporation, but not the evaporation rate for water in the early stages of evaporation. Prediction of the separation distance between the pinned film and the bulk fluid during evaporation remains a challenge. The separation distance is dependent on the depth and width of the channel. Smaller channels were observed to have larger separation distances, which indicate a higher surface tension in smaller channels. This is due to an increase in the capillary force in smaller channels. The ratio of capillary to viscous forces also increases in smaller channels.

DI-water tends to maintain its width for about half of the time of the evaporation cycle (8s), before a significant reduction is observed. The effects of surface tension of water and the hydrophobic nature of the channel walls are factors that explain this deviation from the analytical model. Toluene is completely wetting and it has a lower surface tension, hence it evaporates faster than DI-water. The total evaporation of the bulk fluid occurs in about 3.5s, but the de-pinning process takes a longer time as a result of the strong adhesive force between the pinned film and substrate. This is a result of the wetting effects of the surface on the liquid. The adhesive force between the liquid and substrate increase significantly for the pinned film. This can be attributed to the surface roughness, as the film is enclosed within the rough surface. The roughness height increases the surface area exposed the pinned film. 
The effects of the height of the microchannel were also investigated and reported in Figs. 9 and 10. The change of height of the channel for a fixed width can be associated with a change in volume of the droplet. The mass transfer of the droplet is not significantly affected by a change in depth of the channel as shown in Figs. 9 and 10. A reduction of the height by a third of its original height resulted in a 1s decrease in the evaporation time. This indicates that a change in height of the channel does not have a significant effect on evaporation in the rectangular channel. The surface area of the channel exposed to the atmosphere has a more significant effect on the evaporation rate. The diffusion of the fluid occurs at the surface in contact with air and directly in contact with the fluid. The evaporation rate can be modified by controlling the relative humidity in the vicinity of the ambient air surrounding the channel.

However, the evaporation process did not appear to have a significant effect on the pinning. This occurs due to the separation distance between the bulk fluid and the pinned film as shown in Fig. 6(a). Surface effects have a significant role on the pinned film as a result of the decrease in microchannel depth. This can be attributed to surface tension forces that are relatively larger in the smaller channel, as the capillary force is inversely proportional to the depth of the channel. A reasonable agreement can be observed from the experimental and predicted results in Fig. 10.

The bulk fluid evaporation contact angle varies between 41 and 47 degrees as shown in Fig. 11. The contact angle was measured based on a University of Texas Health Science Center in San Antonio (UTHSCSA) image tool, which uses the pixel displacement to estimate the change in contact angle of both the bulk fluid and pinned film as shown in Fig. 11. The variation in contact angle occurs as a result of the droplet maintaining a nearly constant volume during mass transfer from the droplet surface. The change in angle of the pinned film corresponds to an increase or decrease in the contact angle of the bulk fluid. The pinned film angle varies between 40 and 47 
degrees. The larger channels have a higher rate of evaporation initially due to the surface area of the channel, but after about 7.5s, the effect of surface area is not significant for the range of time considered.

The contact angle with the surface depends on the nature of the surface of the substrate and properties of the channel wall. Although SU-8- is hydrophobic to DI-water, PZT is hydrophilic to DI-water. Toluene has a contact angle of zero since it is completely wetting. The effects of contact angle and channel width on the evaporation rate are reported in Fig. 12. A reduction in contact angle results in a decrease in the evaporation rate of the droplet. Increasing the width increases the evaporation rate of the channel.

Although the channel width has a significant effect on the evaporation of toluene, the experimental results show that it affects the structural layer of the channel. Solid particles are observed along the edges of the channel at the end of the evaporation cycle. During the de-pinning process, once the bulk fluid has evaporated, a split from the center is observed as shown in Fig. 13. The split is different between toluene and water, when comparing the images in Figs. 13 and 14. The difference in wetting properties of toluene and DI-water affects the separation process of the pinned film.

The de-pinning of the pinned toluene film commences immediately after the evaporation of the bulk fluid of toluene in Fig. 13(a). The de-pinning proceeds along the width and length simultaneously as observed in Fig. 13(b). After about 2.67s, the rate of de-pinning is not significant across the length of the channel as observed in Fig. 13(c).The pinned film completely evaporates after about 5.56s as shown in Fig. 13(d). 
De-pinning of DI-water occurs over a longer period of time than toluene. The de-pinning process is significant across the width of the channel, but less across the length, as observed in Fig. 14(a). No further de-pinning across the length can be observed after about 7.24s, as shown in Fig. 14(b). The rate of de-pinning is significantly increased across the width after about 9.04s (Fig. 14(c)). The pinned film completely evaporates after about 10.5s, as the PZT substrate is completely visible across the length and width of the microchannel, as shown in Fig. 14(d). The nature of the PZT substrate surface can be observed after the evaporation and de-pinning processes are complete, as the images are compared for both processes.

A comparison between the de-pinning process for water and toluene is shown in Fig. 15. A reasonable agreement can be observed between the experimental and analytical results. The analytical model accurately predicts the initial start and end of the de-pinning process, but a difference is observed with experimental results mid-way through the de-pinning process. This may have occurred since heat transfer irreversibilities were not fully modelled in the evaporation of the droplet. The experimental measurements were taken when a clear path across the channel could be observed, signifying that mixing of two processes was not occurring simultaneously. The de-pinning rate of toluene was faster than DI-water.

The de-pinning of toluene is multi-dimensional since it occurs in both the axial and transverse directions simultaneously. Only the de-pinning in the transverse direction (across the width) was considered in this study, as the rate is faster in this direction. The length of the channel is significantly larger than the width, hence the mass transfer rate is higher. A numerical twodimensional solution is required for the de-pinning in both directions, however this element was beyond the scope of the present study. 
Strong adhesion was indicated between the pinned film and PZT substrate, as a result of surface effects. The effect of depth of the channel of the substrate on the de-pinning process was also investigated for toluene and reported in Fig. 16. A non-dimensional analysis of analytical results showed a slight difference in the de-pinning rate of about $10 \%$ for the range of depths. The experimental results showed a similar difference at various times. Discrepancies arose due to neglecting de-pinning in the transverse direction in the current model and also neglecting the temperature gradients. Bonn [5] also indicated the complexity of these phenomena remained a challenge in accurately predicting the de-pinning process. The general trend of analytical results is in good agreement with the experimental results. The change in contact line elastic force has an effect on the de-pinning rate [5]. This force increases with defects on the surface, confirming the relationship between surface roughness and de-pinning rate. Further studies would be required to determine the actual effect of the overall volume change on the de-pinning process.

Analytical studies were also investigated to study the effect of channel width on the depinning process. Fig. 17 indicates that larger channels have a higher de-pinning rate than smaller channels. This is expected and experimental results show similar trends. Although this model does not take into account the temperature variations, which occur during mass transfer from the surface, it predicts the experimental trends with reasonable accuracy. Developing a technique to seal the droplet in the MHE will require better understanding of evaporation of the droplet in the microchannel. The ability to control the evaporation process is essential for the successful operation of the MHE. The contact angle can be varied by changing the fluid, substrate or structural layer. In this study, the choice of fluid was based on the ratio of the capillary to viscous forces. A high ratio results in an increase of the bulk velocity for the same amount of heat input. 


\section{$\underline{\text { 5. Conclusions }}$}

This paper examined the use of a PZT substrate in microfluidic transport of droplets. The evaporation and pinning of a droplet in a closed rectangular microchannel was investigated. The rates of evaporation for toluene and DI-water were reported. New experimental and analytical results have been presented for these droplet transport processes. The evaporation of toluene occurred about twice as fast as water in a rectangular channel. Internal recirculation was observed during the evaporation process. This was confirmed by movement of particles during the evaporation process. The different modes of evaporation and de-pinning were reported. Threedimensional printing and other MEMS applications could benefit from the experimental and analytical results in this study. The results also have relevance in biomedical applications where small particles of solute can be distributed through complex meshes.

\section{Appendix A - Solution of mass transfer equation}

Non-dimensionalizing equation (1), the mass transfer equation becomes

$$
\frac{\partial Y}{\partial t}=D_{A B} \frac{\partial^{2} Y}{\partial w^{2}}
$$

The solution of this partial differential equation can be obtained by separation of variables as follows:

$$
\mathrm{Y}=\left[c_{1} \cos (\lambda w)+c_{2} \sin (\lambda w)\right] e^{-D_{A B} \lambda^{2} t}
$$

Applying the boundary conditions will yield the following solution:

$$
\mathrm{Y}=\frac{4 \mathrm{Y}_{0}}{(2 \mathrm{n}+1) \pi} \sum_{\mathrm{n}=0}^{\infty}[\sin (\lambda w)] e^{-D_{A B}\left(\frac{2 n+1}{L}\right)^{2} t}
$$




\section{Acknowledgements}

Financial support from the Canada Research Chairs program and the Natural Sciences and Engineering Research Council of Canada (NSERC) is gratefully acknowledged. The support of the members of the Centre of Integrated Radio Frequency Engineering (CIRFE), University of Waterloo, Waterloo, ON, is also acknowledged.

\section{References}

[1] P.S. Glockner, G.F. Naterer, Surface tension and frictional resistance of thermocapillary pumping in a closed microchannel, International Journal of Heat and Mass Transfer, 49 (2006) 4424-4436.

[2] P.S. Glockner, G.F. Naterer, Thermocapillary control of microfluidic transport with a stationary cyclic heat source, Journal of Micromechanics and Microengineering, 15 (2005) 22162229.

[3] A. Odukoya, G.F. Naterer, I. Dincer, Entropy generation of droplet motion with surface tension hysteresis in a closed microchannel, ournal of Micromechanics and Microengineering, 21 (2011) (in press).

[4] P.S. Glockner, G.F. Naterer, Interfacial thermocapillary pressure of an accelerated droplet in microchannels: Part I. Fluid flow formulation, International Journal of Heat and Mass Transfer, 50 (2007) 5269-5282.

[5] D. Bonn, J. Eggers, J. Indekeu, J. Meunier, E. Rolley, Wetting and spreading, Reviews of modern physics, 81 (2009) 739. 
[6] K. Ragil, D. Bonn, D. Broseta, J. Meunier, Wetting of alkanes on water from a Cahn-type theory, Journal of Chemical Physics, 105 (1996) 5160-5167.

[7] G. McHale, S.M. Rowan, M.I. Newton, M.K. Banerjee, Evaporation and the wetting of a lowenergy solid surface, Journal of Physical Chemistry B, 102 (1998) 1964-1967.

[8] M. Cachile, O. Benichou, A. Cazabat, Evaporating droplets of completely wetting liquids, Langmuir, 18 (2002) 7985-7990.

[9] S.M. Rowan, M.I. Newton, G. McHale, Evaporation of microdroplets and the wetting of solid surfaces, Journal of Physical Chemistry, 99 (1995) 13268-13268.

[10] R.D. Deegan, Pattern formation in drying drops, Physical Review E (Statistical Physics, Plasmas, Fluids, and Related Interdisciplinary Topics), 61 (2000) 475-485.

[11] C. Poulard, G. Guéna, A.M. Cazabat, A. Boudaoud, M.B. Amar, Rescaling the dynamics of evaporating drops, Langmuir, 21 (2005) 8226-8233.

[12] N. Shahidzadeh-Bonn, S. Rafai, A. Azouni, D. Bonn, Evaporating droplets, Journal of Fluid Mechanics, 549 (2006) 307-313.

[13] H. Hu, R.G. Larson, Evaporation of a sessile droplet on a substrate, Journal of Physical Chemistry B, 106 (2002) 1334-1344.

[14] R.D. Deegan, O. Bakajin, T.F. Dupont, G. Huber, S.R. Nagel, T.A. Witten, Contact line deposits in an evaporating drop, Physical Review E, 62 (2000) 756. 
[15] R.D. Deegan, O. Bakajin, T.F. Dupont, Capillary flow as the cause of ring stains from dried liquid drops, Nature, 389 (1997) 827-829.

[16] J. Fukai, H. Ishizuka, Y. Sakai, M. Kaneda, M. Morita, A. Takahara, Effects of droplet size and solute concentration on drying process of polymer solution droplets deposited on homogeneous surfaces, International Journal of Heat and Mass Transfer, 49 (2006) 3561-3567.

[17] J.R. Welty, C.E. Wicks, G. Rorrer, R.E. Wilson, Fundamentals of Momentum, Heat, and Mass Transfer, Wiley-India, 2009.

[18] S.H. Davis, Thermocapillary Instabilities, in: Annual Reviews Inc, Palo Alto, CA, USA, 1987, pp. 403-435.

[19] E. Sultan, A. Boudaoud, M. Ben Amar, Diffusion-limited evaporation of thin polar liquid films, Journal of Engineering Mathematics, 50 (2004) 209-222.

[20] C. Bourges, M.E.R. Shanahan, Influence of evaporation on the contact angle of water drops, Comptes Rendus de l'Academie des Sciences, Serie II (Mecanique, Physique, Chimie Sciences de la Terre et de l'Univers). 316 (1993) 311-316.

[21] D. Bonn, J. Meunier, Comment on evaporation preempts complete wetting, Europhysics Letters, 39 (1997) 341-242.

[22] Z. Pan, H. Wang, Symmetry-to-asymmetry transition of Marangoni flow at a convex volatizing meniscus, Microfluid Nanofluid. 9 (2010) 657-669. 
[23] S.J. Kline, F. McClintock, Describing uncertainties in single-sample experiments, Mechanical Engineering, 75 (1953) 3-8. 


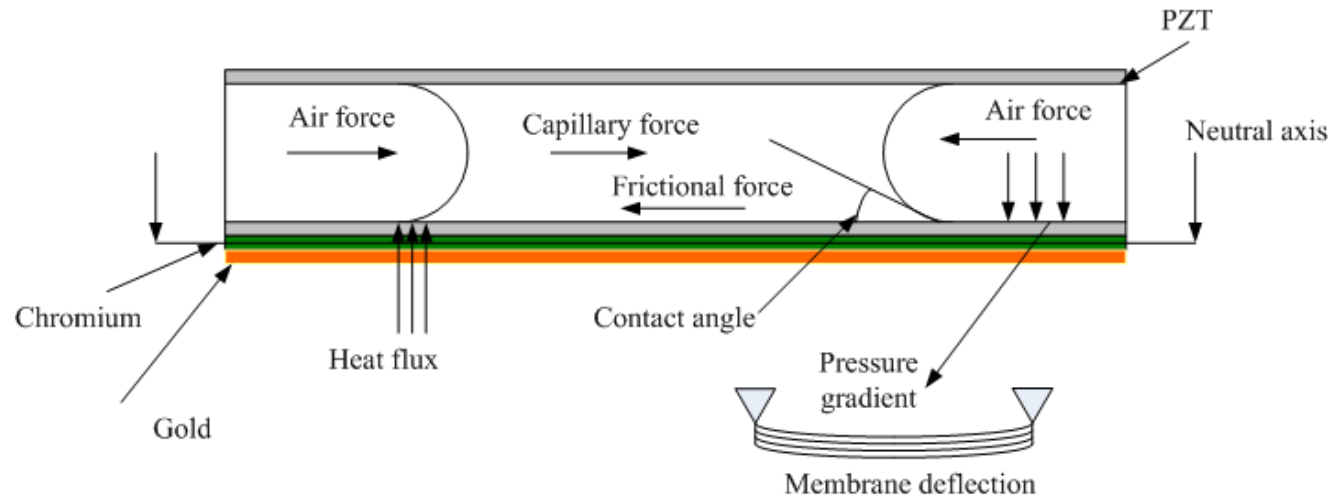

Fig. 1: Schematic of problem configuration in the MHE

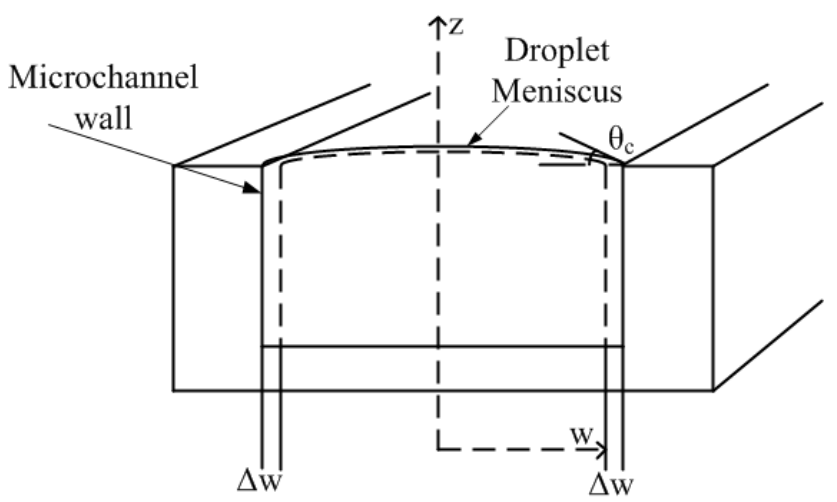

Fig. 2: Schematic of the evaporation process in a closed rectangular microchannel. The change in droplet width for either end of the channel is $\Delta \mathrm{w}$. The model represents half of the channel width. The contact angle $\theta_{c}$ is the angle made by the bulk fluid with the surface of the channel. 


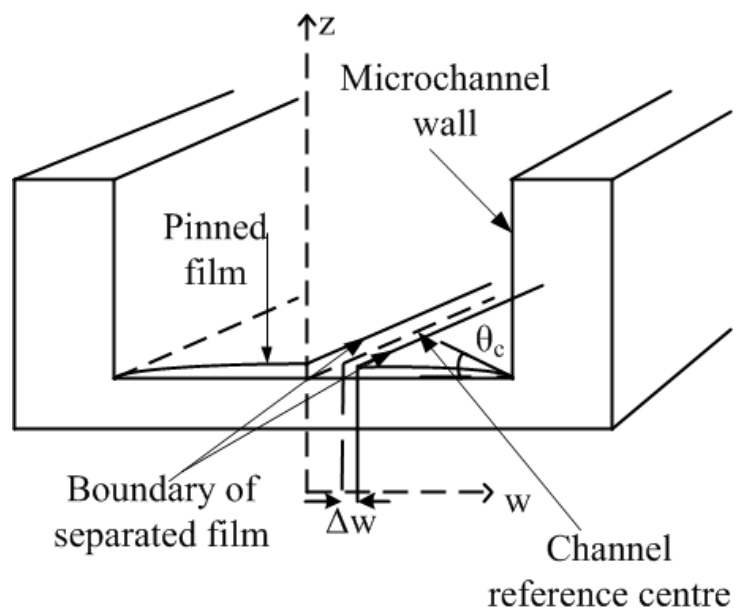

Fig. 3: Schematic of the de-pinning process in a closed rectangular microchannel, where the change in width of the film is $\Delta \mathrm{w}$. The depinning process proceeds in both directions. The contact angle $\theta_{c}$ is the measured angle made by the pinned film with the surface of the channel.

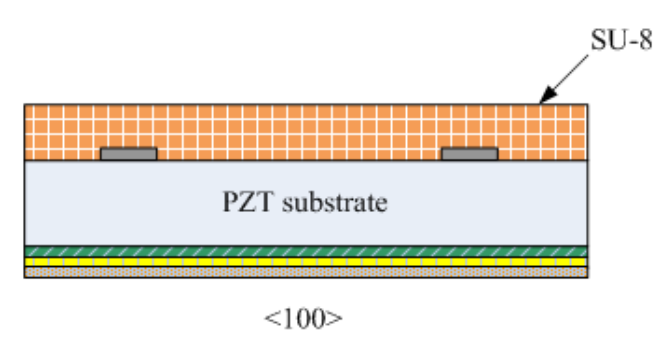

(i)

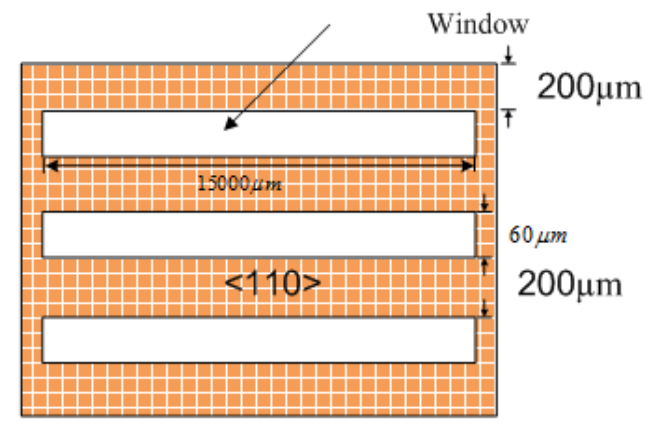

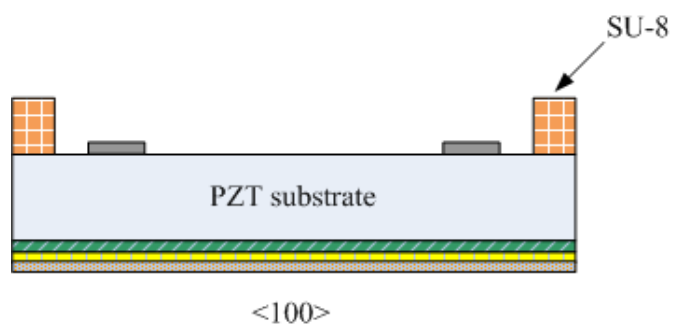

(j)

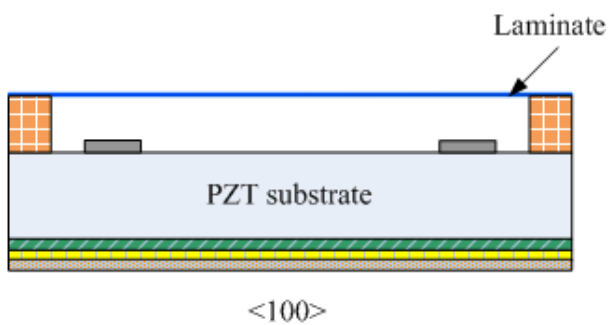

(k)

Fig. 4: Fabrication of SU-8 microchannels using photolithography and sealing of the microchannel with a Bungard dry film laminator. (a) Deposition of SU-8 on PZT subtrate and mask used to pattern the SU-8 channel during photolithography. (b) SU-8 channel after wet etching of a patterned surface. (c) Laminted MHE with Bunduard film laminator. 


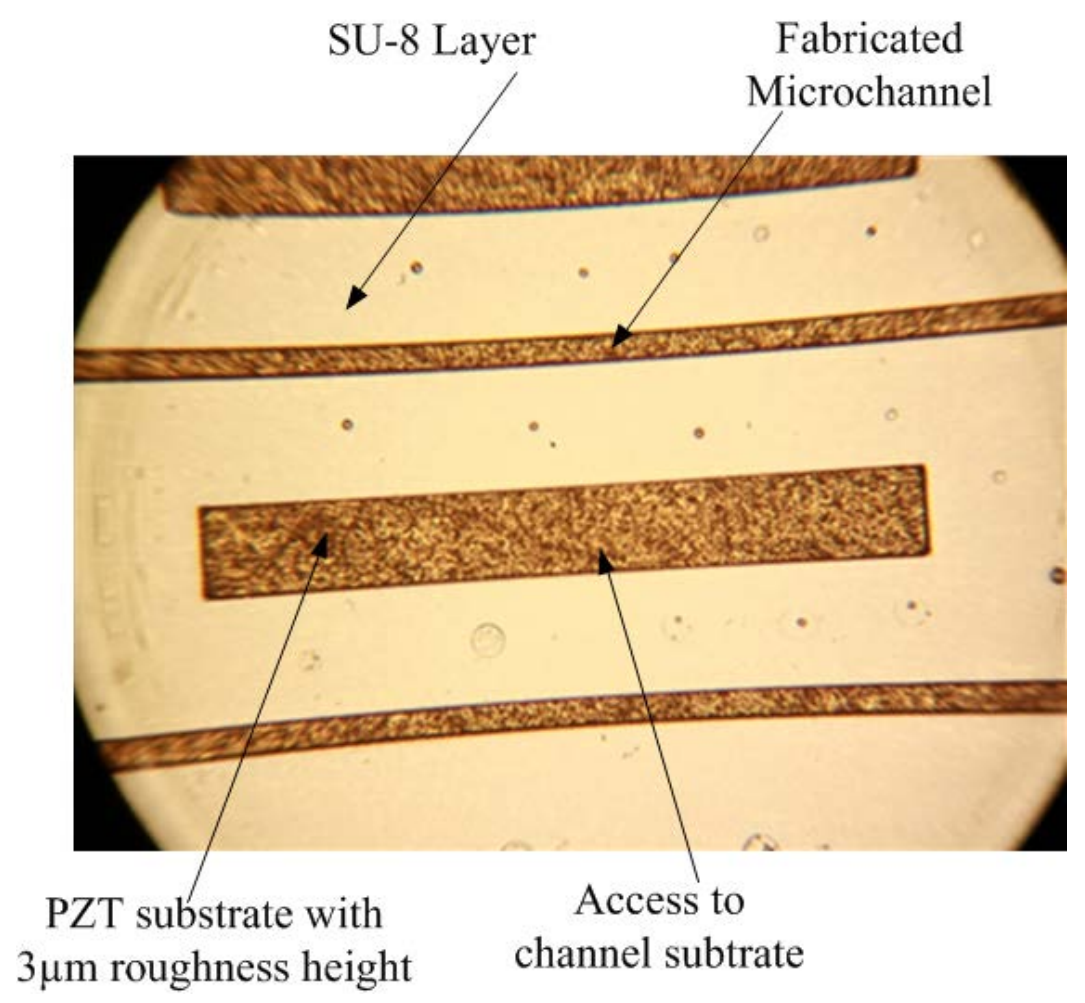

Fig. 5: Fabricated SU-8 2075 80 $\mu$ m microchannel viewed at 5× magnification of a Reichert Austria stereo microscope.

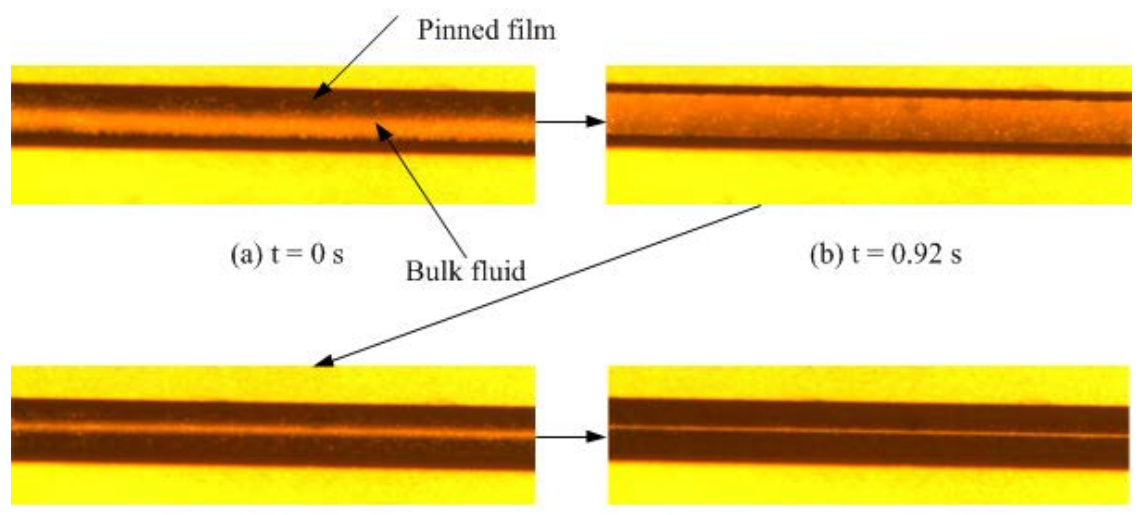
(c) $\mathrm{t}=3.32 \mathrm{~s}$
(d) $\mathrm{t}=6.08 \mathrm{~s}$

Fig. 6: (a) Initial separation of a DI-water droplet and pinned film at time $t=0 \mathrm{~s}$. (b) Maximum spreading of the bulk fluid at time $t=0.92 \mathrm{~s}$. (c) Reduction in evaporation rate at time $\mathrm{t}=3.32 \mathrm{~s}$. (d) End of the evaporation of the bulk fluid at time $t=6.08 \mathrm{~s}$ (Magnification is $\times 5$ ). 

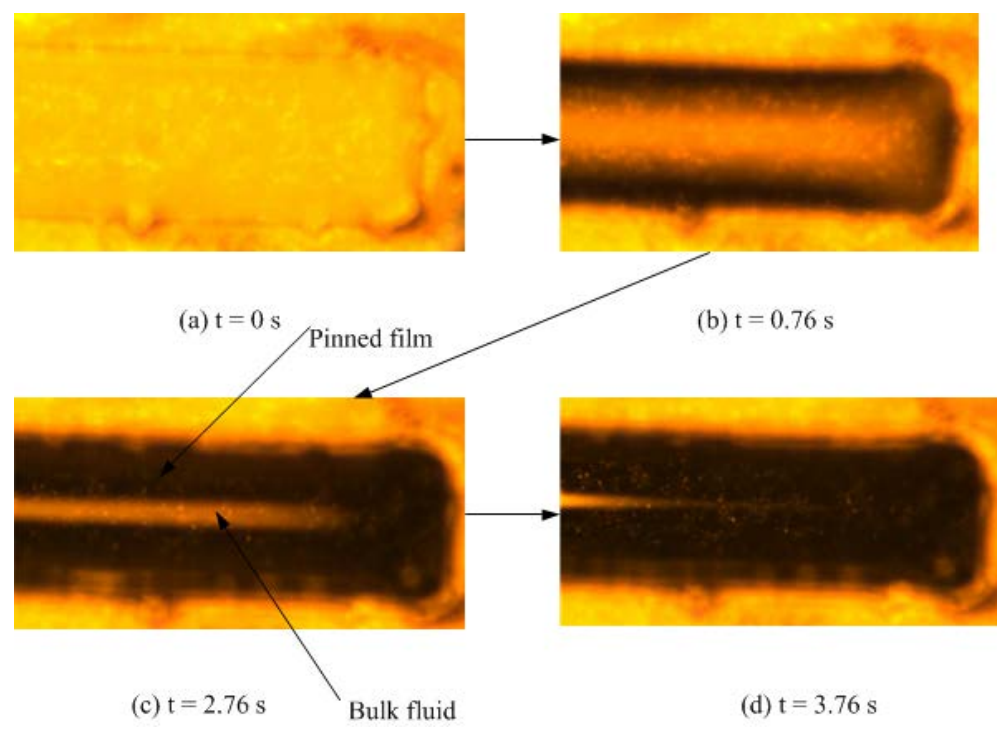

Fig. 7: (a) Initial wetting of toluene on a substrate at time $t=0 \mathrm{~s}$. (b) Beginning of separation of a bulk fluid and pinned film at time $t=0.76 \mathrm{~s}$. (c) Reduction in the evaporation rate at time $\mathrm{t}=2.76 \mathrm{~s}$.

(d) End of the evaporation of a bulk fluid at time $t=3.76$ (Magnification is $\times 10$ ).

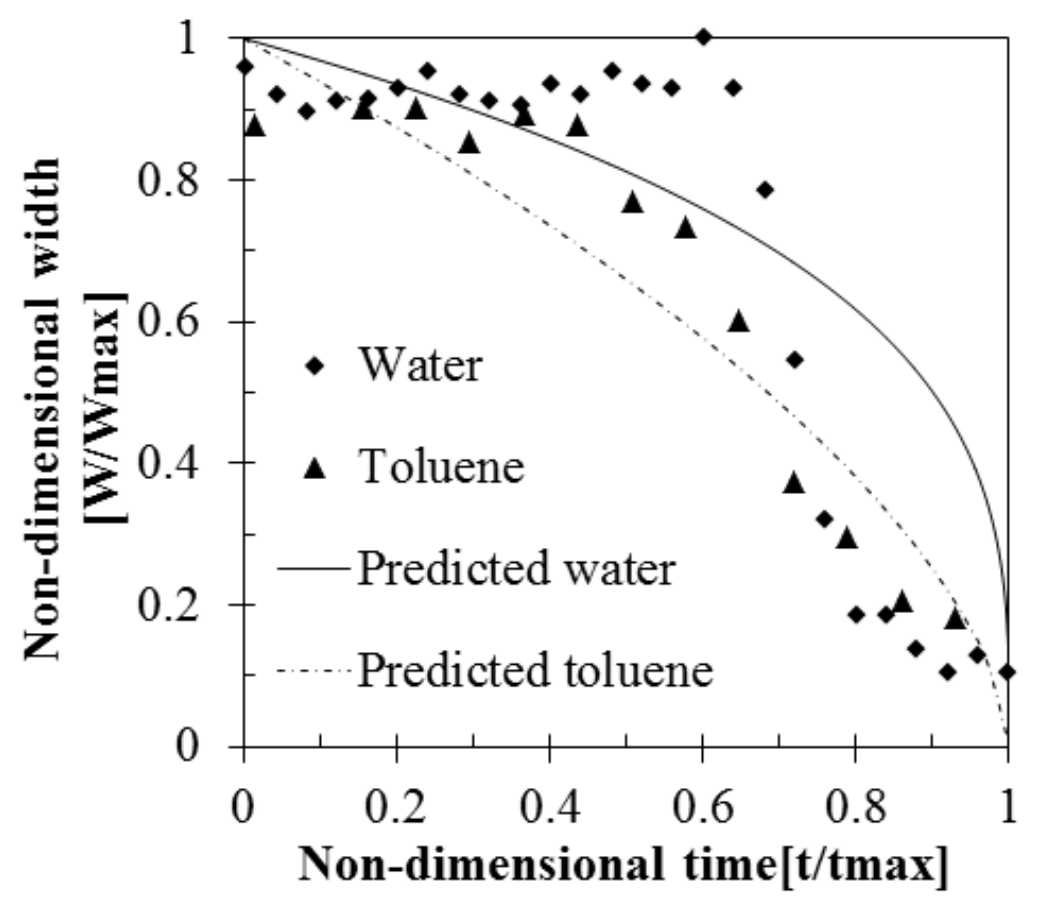

Fig. 8: Comparison of experimental and analytical results of evaporation in the microchannel 


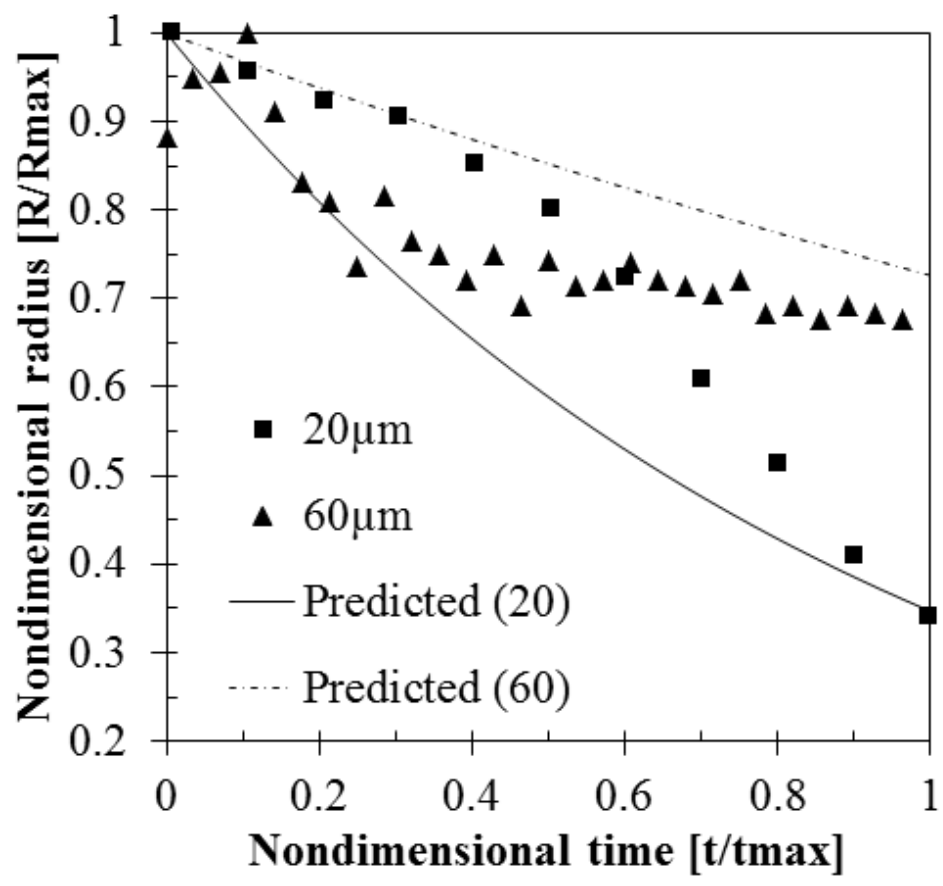

Fig. 9: Comparison of experimental and analytical results of evaporation of DI-water in a rectangular microchannel of different depths

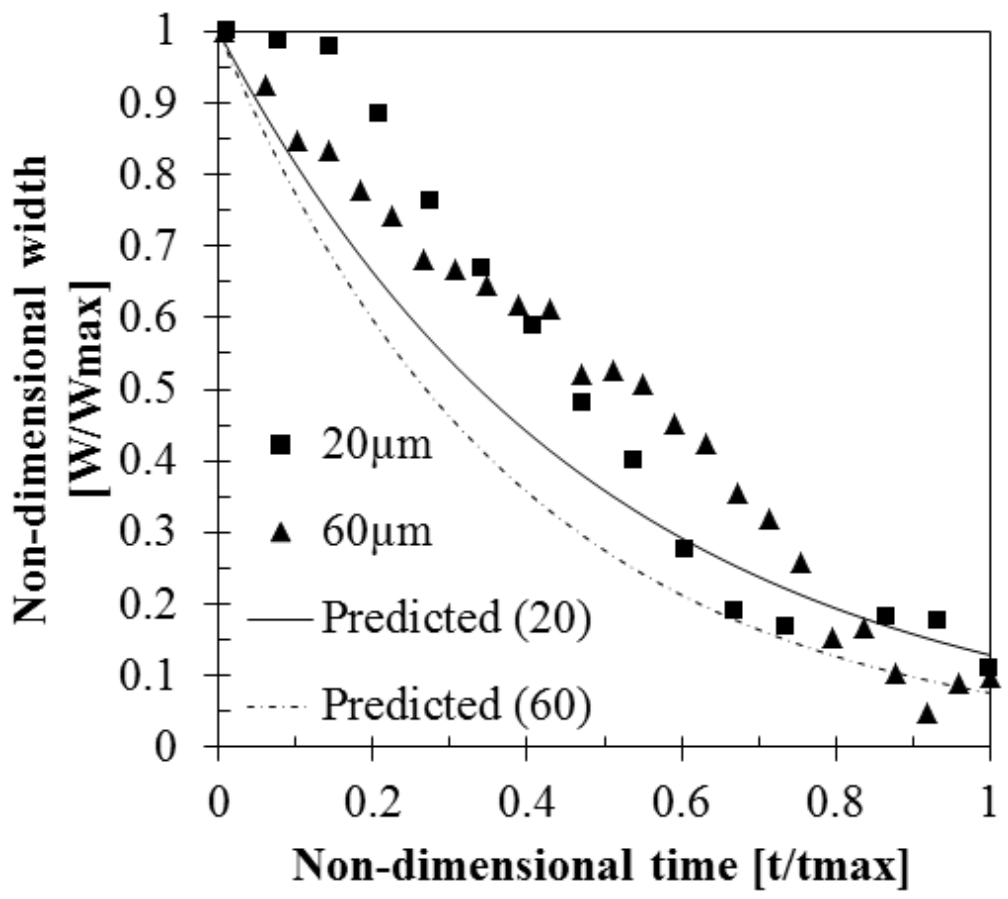

Fig. 10: Comparison of experimental and analytical results of evaporation of toluene in a rectangular microchannel of different depths. 


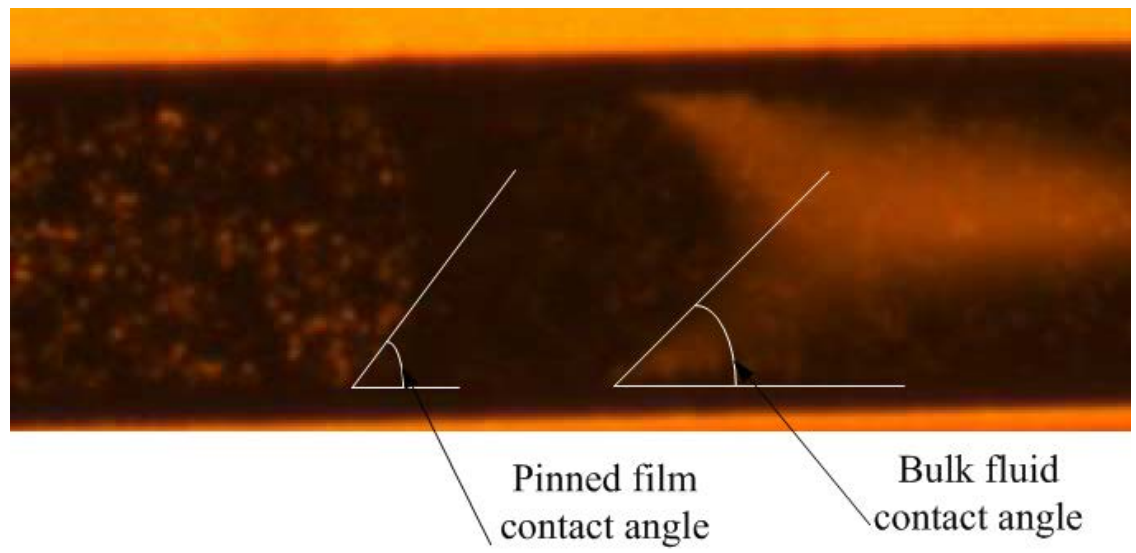

Fig. 11: Bulk and pinned film contact angle measurement using UTHSCSA image tool.

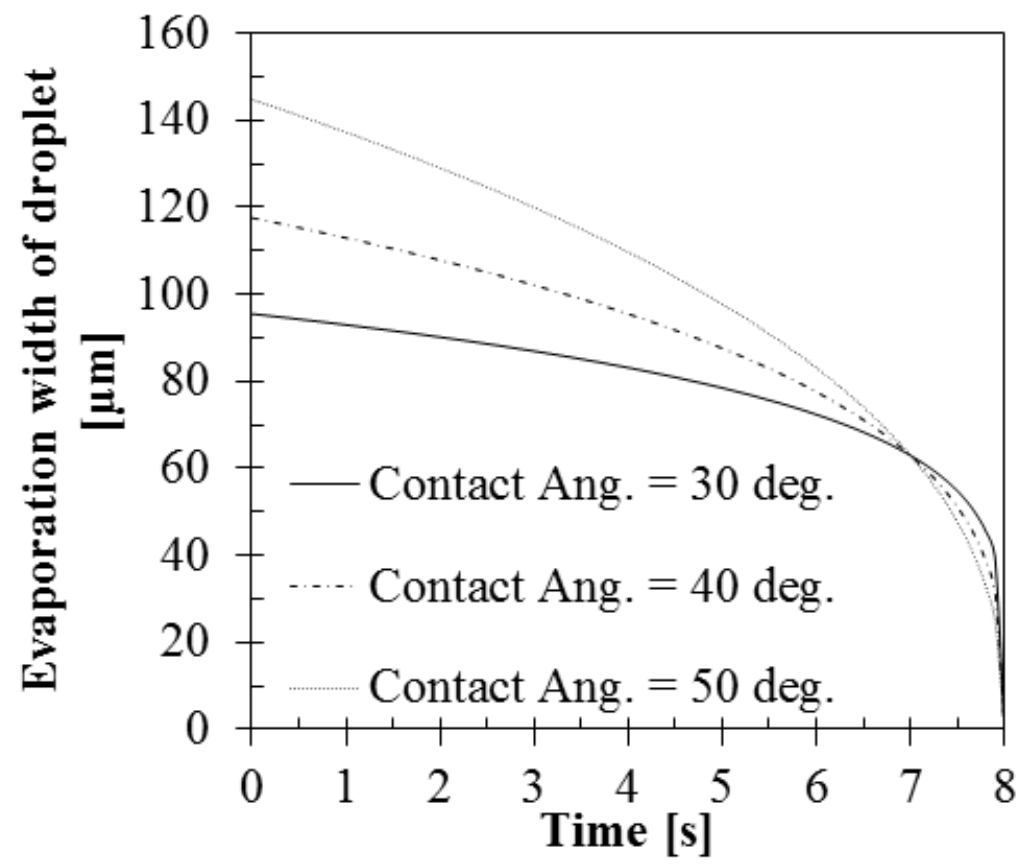

Fig. 12: Effects of contact angle and width on evaporation of the bulk fluid 

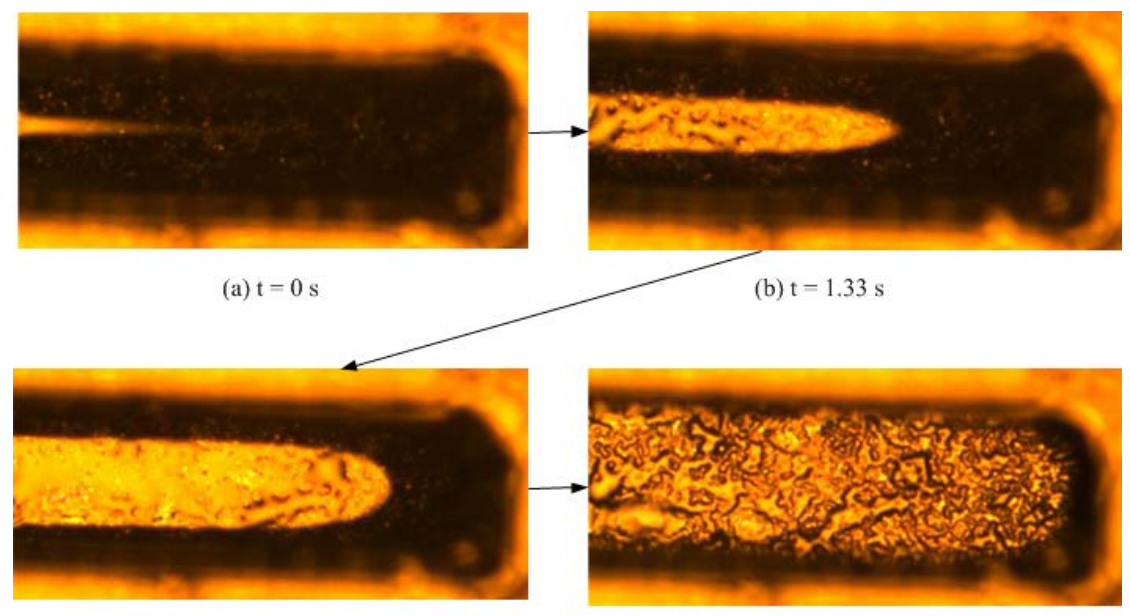

(c) $\mathrm{t}=2.67 \mathrm{~s}$

(d) $\mathrm{t}=5.56 \mathrm{~s}$

Fig. 13: Initial de-pinning of toluene droplet after evaporation of bulk fluid at time $t=0$ s. (b) Increase in de-pinning rate across the length and width of the droplet simultaneously at time $t=1.33 s$. (c) Decrease in de-pinning rate across the length of the droplet at time $t=2.67 \mathrm{~s}$. (d) End of de-pinning at time $t=5.56$ s (Magnification is $\times 10$ ).

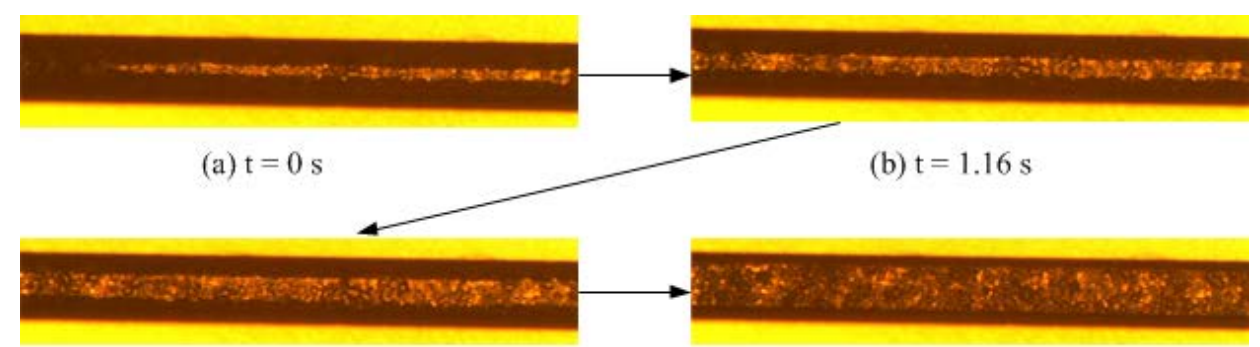

(c) $\mathrm{t}=2.96 \mathrm{~s}$

(d) $\mathrm{t}=4.42 \mathrm{~s}$

Fig. 14: Initial de-pinning of DI-water droplet after evaporation of bulk fluid at time $t=0$ s. (b) End of simultaneous de-pinning across the length and width of the film with maximum spreading of the bulk fluid at time $t=1.16$ s. (c) Significant increase in de-pinning rate at time $t=2.96 \mathrm{~s}$. (d) End of de-pinning at time $t=4.42 \mathrm{~s}$ (Magnification is $\times 5$ ). 


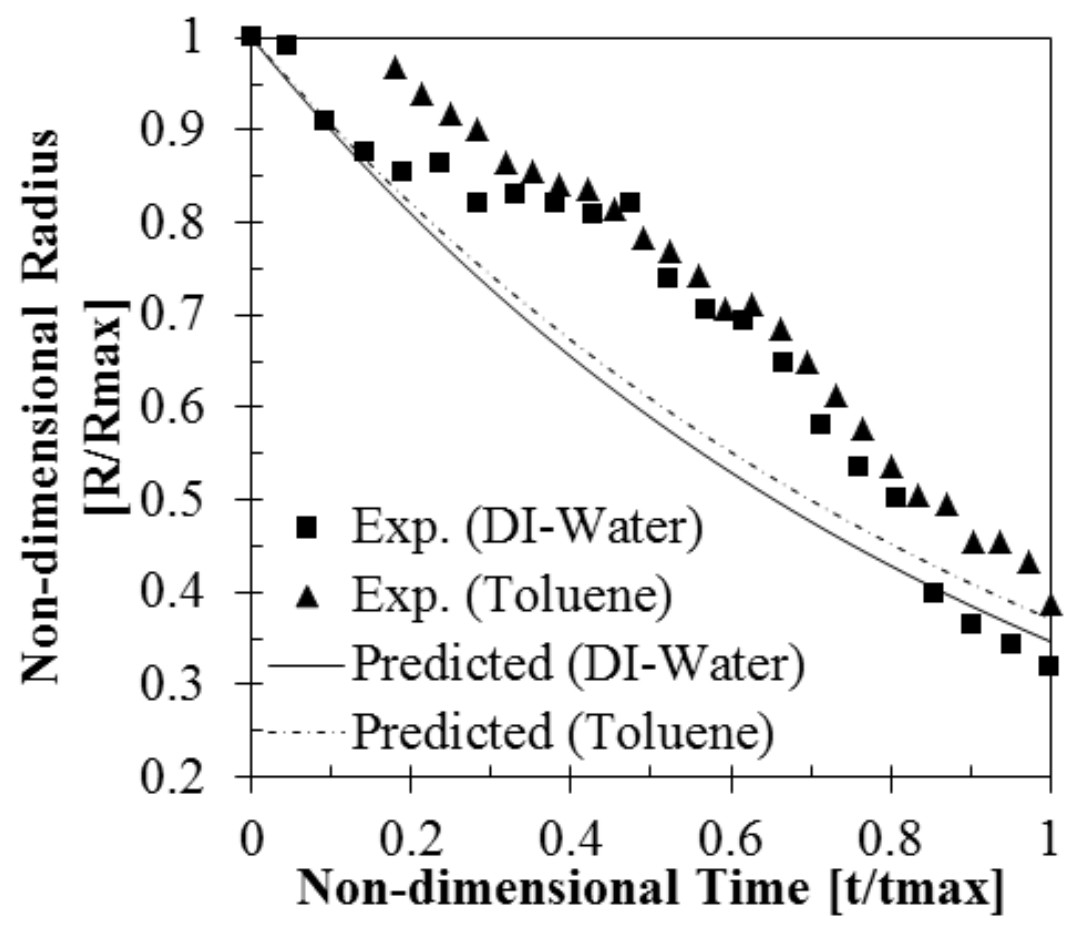

Fig. 15: Comparison of experimental measurements and analytical predictions of de-pinning

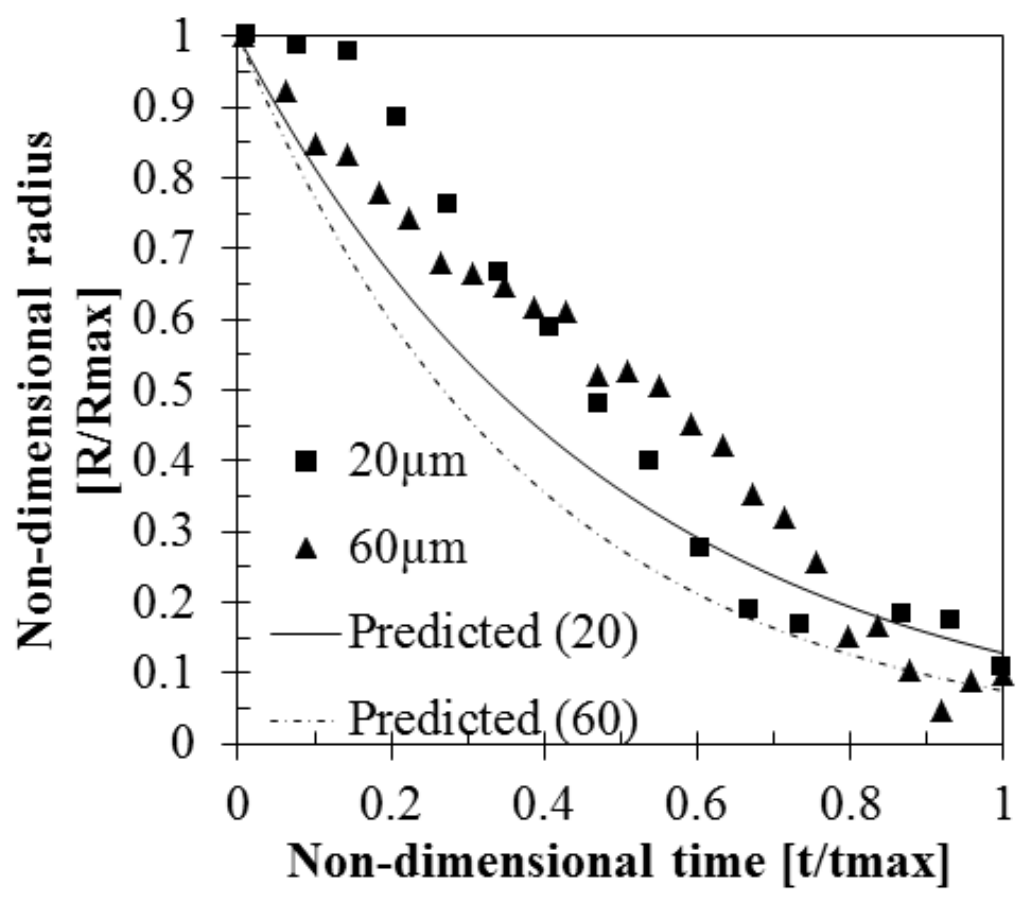

Fig. 16: Comparison of experimental and analytical results of de-pinning of toluene 


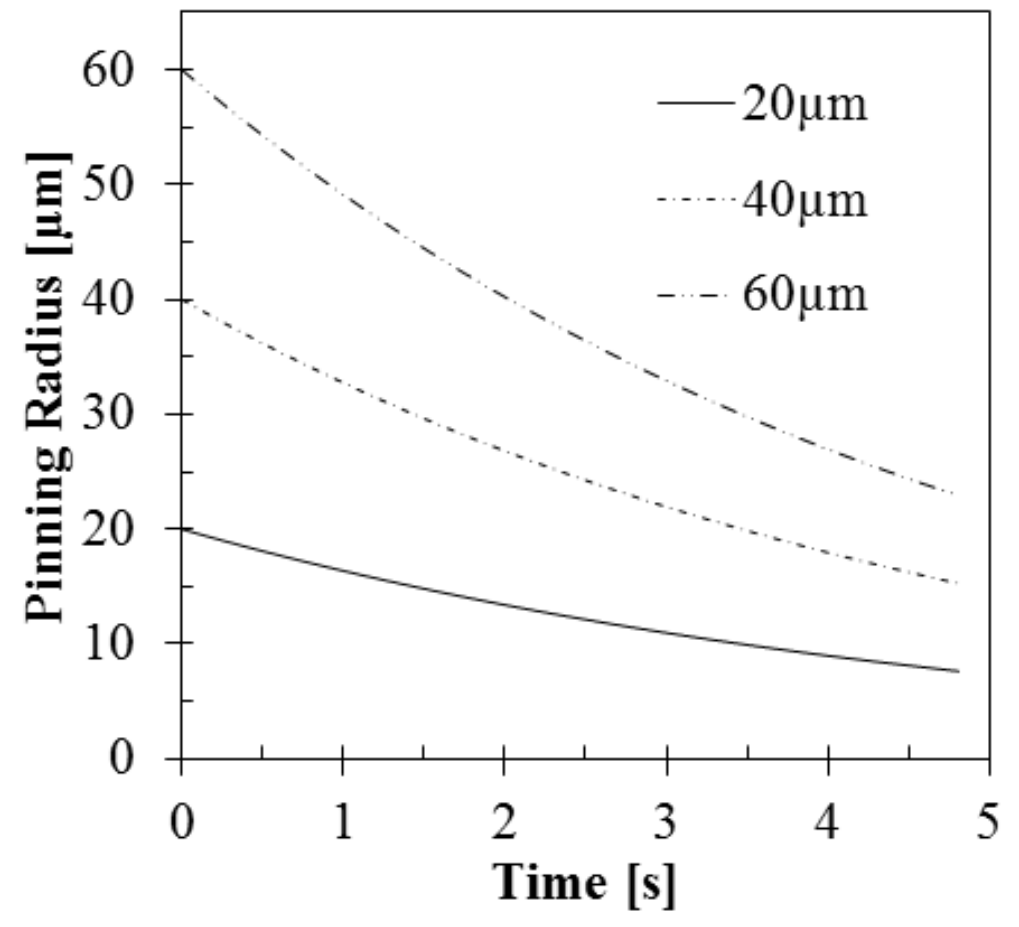

Fig. 17: Effect of microchannel width on de-pinning of the bulk fluid 